\title{
Rotaxane-based molecular machines operated by photoinduced electron transfer
}

\author{
Alberto Credi* and Belén Ferrer \\ Dipartimento di Chimica "G. Ciamician", Università di Bologna, via Selmi 2, \\ 40126 Bologna, Italy
}

\begin{abstract}
A molecular machine is an assembly of a definite number of molecular components designed to perform mechanical motions as a result of an appropriate external stimulation. Like their macroscopic counterparts, nanoscale machines need energy to operate. Energy can be supplied through (i) chemical reactions, (ii) electrochemical processes, and (iii) photoinduced processes. Although most molecular motors of the biological world are fueled by chemical reactions, for several reasons light is a very good choice to operate artificial molecular machines. Rotaxanes, owing to their peculiar architecture, are attractive candidates for the construction of artificial nanoscale machines. By adopting an incrementally staged design strategy, photoinduced electron transfer processes have been engineered within rotaxane-type structures with the purpose of obtaining light-powered molecular machines. Such an approach is illustrated by describing the behavior of prototypes investigated in our laboratories.
\end{abstract}

\section{INTRODUCTION}

Our body can be viewed as a very complex ensemble of molecular machines that power our motions, repair damage, and orchestrate our inner worlds of sense, emotion, and thought [1]. The operation of several of such biomotor molecules is well understood [2]. The idea of constructing artificial molecular machines, however, is rather recent [3], and only in the last 10 years systematic studies have been performed in this field $[4,5]$.

A molecular machine can be defined [4] as an assembly of a discrete number of molecular components (that is, a supramolecular structure) designed to perform mechanical-like movements as a consequence of appropriate external stimuli. The extension of the concept of machine to the molecular level is important not only for the sake of basic research, but also for the growth of nanoscience and the development of a bottom-up approach to nanotechnology. Chemists, by the nature of their discipline, are already at the bottom, since they are able to manipulate molecules (i.e., the smallest entities with distinct shapes and properties) and are therefore in the ideal position to develop bottom-up strategies for the construction of nanoscale machines.

Molecular machines operate via electronic and nuclear rearrangements, that is, through some kind of chemical reaction. Like macroscopic machines, they are characterized by (i) the kind of energy input supplied to make them work, (ii) the type of movement performed by their components, (iii) the way in which their operation can be monitored, (iv) the possibility to repeat the operation at will, thus establishing a cyclic process, (v) the time scale needed to complete a cycle of operation, and (vi) the function that can be ultimately carried out. 
Of the above list, point (i) - namely, the energy supply of the molecular machine - is certainly the most significant. The obvious way to provide a chemical system with energy is through an exergonic chemical reaction. This is what happens in our body, where the chemical energy supplied by food is stored in ATP molecules and used to power the biological machines that sustain life [2]. If a molecular machine has to work by inputs of chemical energy, it will need addition of fresh reactants ('fuel') at any step of its working cycle, with the concomitant formation of waste products. Accumulation of such waste products, however, will compromise the cyclic operation of the device unless they are removed from the system, as it happens in our body as well as in macroscopic internal combustion engines. In any case, since a molecular machine has to work by repeating cycles \{point (iv)\}, a fundamental requirement is that any chemical process taking place in the system has to be reversible.

Chemical fuel is not the only means by which energy can be supplied to operate molecular machines. Nature shows that in green plants the energy needed to sustain the machinery of life is supplied by sunlight. Photochemical energy inputs can indeed cause the occurrence of endergonic chemical reactions, which can make a machine work without formation of waste products. There is currently an increasing interest in the development of photon-powered molecular devices and machines, taking advantage of the recent, outstanding progresses made by supramolecular photochemistry [6]. Photochemical inputs offer other advantages compared to other types of energy inputs. For example, they can be switched on and off easily and rapidly, and lasers provide the opportunity of working in small space and very short time domains. It should also be noted that light energy can be supplied to a molecule without physically 'touching' it, whereas in order to make use of electrical energy a chemical system needs to be connected to a circuit (e.g., an electrochemical cell).

In order to control and monitor the machine operation \{point (iii)\}, the motion of the component parts should cause readable changes in some chemical or physical property of the system. Photochemical methods are very useful in this regard since photons are at the same time quanta of energy and bits of information and can play the dual role of 'writing' (i. e., causing a change in the system) and 'reading' (i. e., reporting the state of the system). Luminescence spectroscopy, in particular, is a valuable technique since it is easily accessible and offers good sensitivity and selectivity, along with the possibility of time-resolved studies.

\section{PSEUDOROTAXANES AND ROTAXANES AS MOLECULAR MACHINES}

Pseudorotaxanes are host/guest systems composed minimally of a thread-like molecule surrounded by a macrocycle (Fig. 1a). The presence of bulky substituents at the extremities of the thread component that prevent dissociation of the two molecular components leads to rotaxanes (Fig. 1b). Dethreading/rethreading of the ring and thread components of a pseudorotaxane (Fig. 1a) and shuttling of the ring along the axle component in a rotaxane (Fig. 1b) are some of the molecular motions that can be obtained with these compounds. In the light of such structural features, it is no surprise that the majority of artificial nanoscale machines developed so far are based on this kind of molecular architectures.

Rational and efficient synthetic approaches for the preparation of pseudorotaxanes and rotaxanes are available [7]. One of such strategies, developed by Stoddart and coworkers, is based on charge-transfer and $\mathrm{C}-\mathrm{H} \cdots \mathrm{O}$ interactions between an electron acceptor like the 4,4'bipyridinium unit and an electron donor like dioxyaromatic groups [8]. The charge-transfer interaction (CT) between electron acceptor and electron donor units not only stabilizes a specific mutual arrangement of the molecular components, but also modifies the physical properties of the assembly in comparison to those of the isolated components. From the spectroscopic viewpoint, the CT interaction introduces low energy excited states that cause the presence of broad and weak absorption bands in the visible region, and the quenching of the potentially luminescent excited states localized on the molecular components [9]. 
a

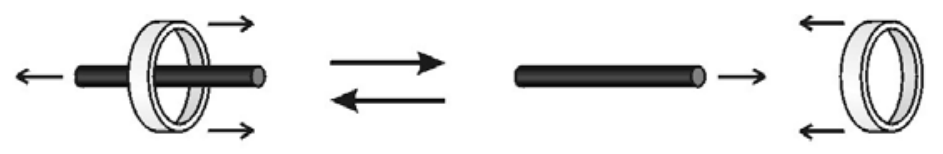

b

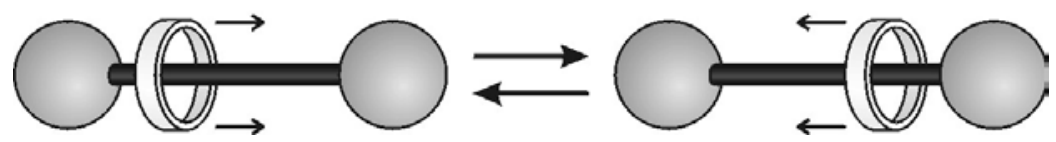

Fig. 1 Representation of machine-like motions that can be obtained in pseudorotaxanes (a) and rotaxanes (b).

It can be expected that in pseudorotaxanes and rotaxanes based on $\mathrm{CT}$ interactions, mechanical movements can be promoted by modulating such interactions. This result can be achieved by reducing the electron acceptor unit(s) or oxidizing the electron donor one(s) by means of chemical, electrochemical or photochemical reactions. Here we will discuss the case of photochemical reactions.

\section{PISTON/CYLINDER SYSTEMS OPERATED BY LIGHT}

Dethreading/rethreading of the wire and ring components of a pseudorotaxane reminds the movement of a piston in a cylinder (Fig. 1a). The first attempts at designing a photochemically driven molecular machine of this type were carried out on the pseudorotaxane $[\mathbf{1} \subset \mathbf{2}]^{4+}$ (Fig. 2), self-assembled in acetonitrile or water solution by the electron donor thread $\mathbf{1}$ and the electron acceptor macrocycle $\mathbf{2}^{4+}$. In such system, light excitation in the visible CT absorption bands leads formally to the transfer of an electron from the donor to the acceptor component. As a consequence, particularly when this process leads to formation of charges of the same sign in the two components, one can expect destabilization of the pseudorotaxane structure followed by dethreading. In practice, however, this simple approach does not work because the back electrontransfer process is much faster than the separation of the molecular components (Fig. 2), a process which requires extended nuclear motions and solvent rearrangement.

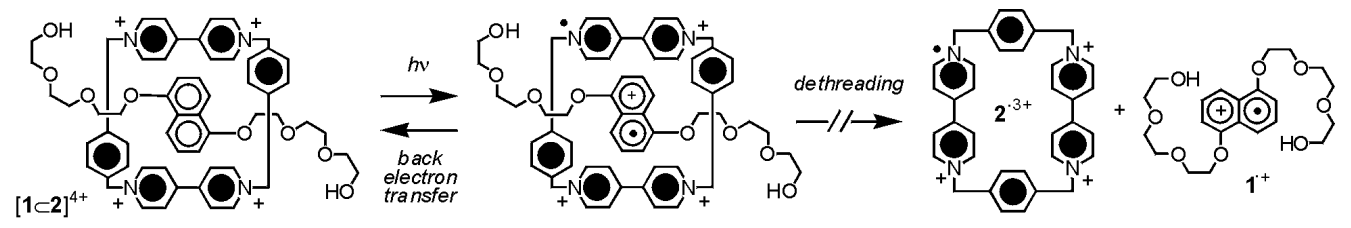

Fig. 2 Reactions associated with the pseudorotaxane $[\mathbf{1} \subset \mathbf{2}]^{4+}$ upon excitation in its $\mathrm{CT}$ absorption band.

In order to achieve photoinduced dethreading, a different approach has been devised [10], based on the use of an external electron-transfer photosensitizer (P) and a sacrificial reductant, as illustrated in Fig. 3. The photosensitizer must be able to (i) absorb light efficiently and (ii) have a sufficiently long-lived and reductant excited state, so that its excitation (process 1) in the presence of the pseudorotaxane will lead (process 2) to the transfer of an electron to a bipyridinium unit of the cyclophane. The relatively fast back electron transfer from the reduced cyclophane component to the oxidized photosensitizer is prevented by the sacrificial reductant which, if present in a sufficient amount, intercepts the oxidized photosensitizer and regenerates (process 3) its original redox state. Good candidates for the role of photosensitizer are 9-anthracenecarboxylic acid and metal complexes such as $\left[\mathrm{Ru}(\mathrm{bpy})_{3}\right]^{2+}$, while efficient reductant scavengers are triethanolamine and polycarboxylate (e.g., oxalate) anions. Under these conditions, the persistent reduction of a bipyridinium unit of $2^{4+}$ is achieved and the pseudorotaxane dethreads (process 4 ), as evidenced by 
absorption spectral changes and by the increase in the intensity of the dioxynaphthalene fluorescence, that can only originate from free 1. Oxygenation of the solution, from which $\mathrm{O}_{2}$ was initially removed, reoxidizes the macrocycle back to the tetracationic form (process 5), thereby promoting rethreading with $\mathbf{1}$ (process 6), as indicated by the absorption and luminescence spectra. It has been shown [11a] that this same system can be operated in the interior pores of a sol-gel silica framework as well as on a solid support by trapping the wire-like molecule onto the surface of a sol-gel film. Similar compounds have been recently used to construct a chemically controllable supramolecular nanovalve [11b].

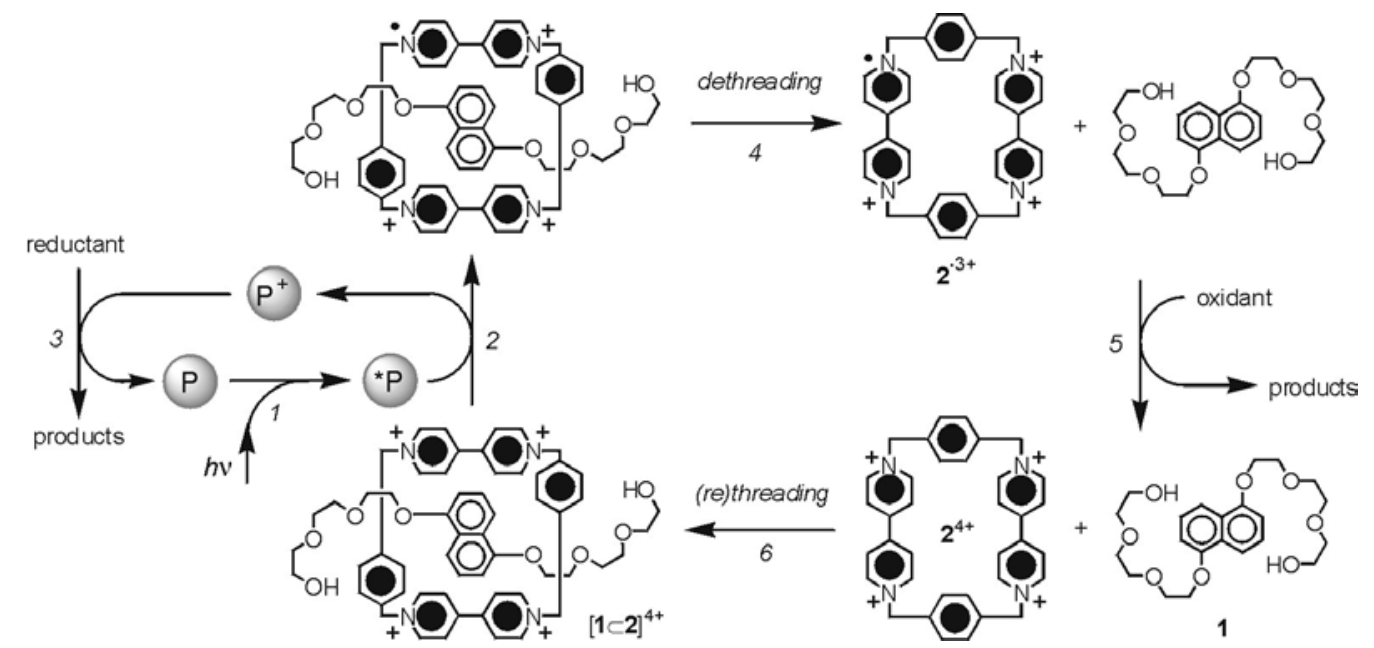

Fig. 3 Photochemically induced dethreading of pseudorotaxane $[\mathbf{1} \subset \mathbf{2}]^{4+}$ based on the use of an external photosensitizer $(\mathrm{P})$ and a reductant scavenger. Rethreading occurs upon addition of an oxidant (e.g., $\mathrm{O}_{2}$ ).
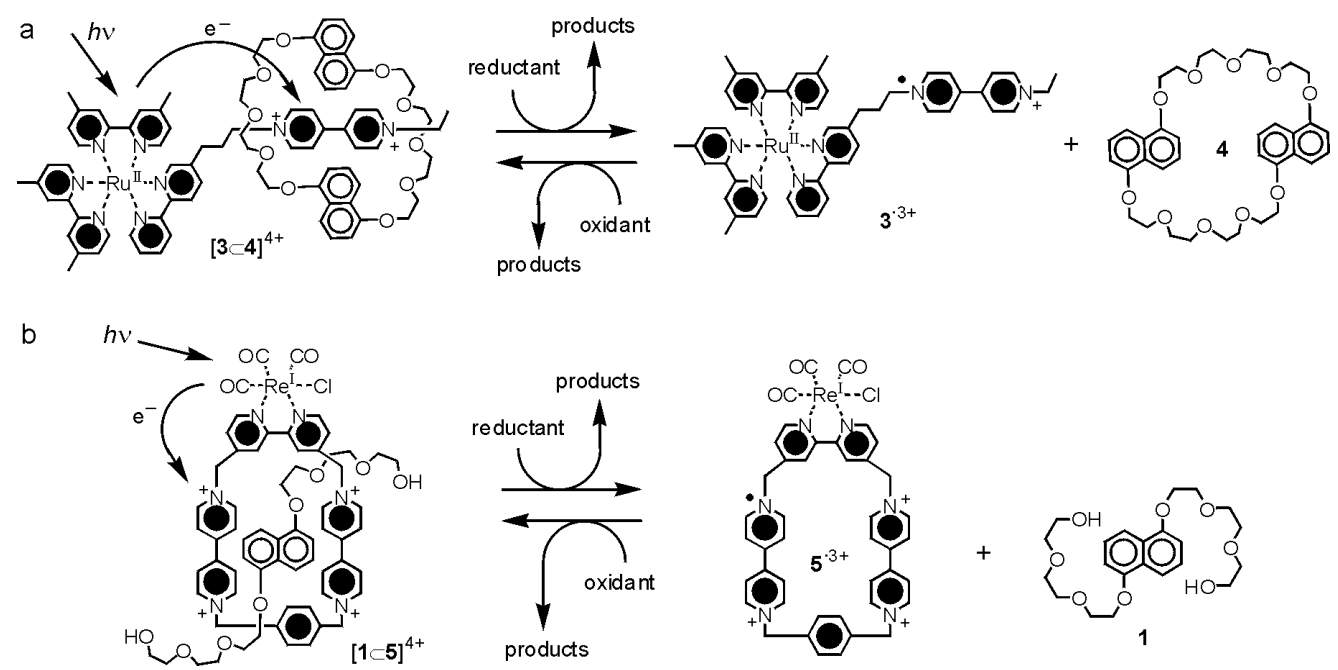

Fig. 4 Second-generation photocontrollable molecular machines based on pseudorotaxanes.

This strategy has been extended recently to second-generation pseudorotaxanes $[3 \subset 4]^{4+}$ and $[\mathbf{1} \subset \mathbf{5}]^{4+}$ in which the metal-complex photosensitizer (the 'light-fueled' motor) has been incorporated either into the thread (Fig. 4a) [12a] or into the ring (Fig. 4b) [12b] component. The 
successful operation of these pseudorotaxanes as molecular machines is the result of (i) the appropriate choice of the functional units and (ii) their covalent linking into the thread and ring components in order to achieve the correct integration of the needed functions (e.g., receptor ability, redox features, photophysical properties, etc.), the right sequence of processes, and the lack of interference between the active units. As in the case of the molecular machine shown in Fig. 3, the dethreading and rethreading motions of the pseudorotaxanes represented in Fig. 4 can be triggered by visible light irradiation and oxygenation of the solution, respectively, and the motions can be monitored by means of UV-Vis absorption and luminescence spectroscopy. The most important readout signal is the intensity of the dioxynaphthalene fluorescence associated with the free ring 4 (Fig. 4a) or free thread 1 (Fig. 4b) components. It is worth noting that many \{deoxygenation-irradiation(dethreading)/oxigenation(rethreading)\} cycles can be performed on the same solution without any appreciable loss of signal until the reductant scavenger is consumed. It should also be stressed that systems which rely on this photosensitizer-scavenger strategy utilize, in addition to light energy, the irreversible decomposition of a reductant scavenger that produces 'waste' species. In this regard, the search for efficient molecular machines exploiting 'clean', reversible photochemical reactions (in other words, machines which use only light as an energy supply) is of fundamental importance.

\section{A PHOTOCHEMICALLY DRIVEN MOLECULAR SHUTTLE}

In a rotaxane with two recognition sites (stations) located on the thread component, the macrocyclic ring can 'shuttle' between the stations (Fig. 1b). If the two recognition sites are chemically different, the motion of the ring can be externally controlled. The design principles at the basis of the light-driven molecular machines shown in Fig. 4 have been employed to obtain the rotaxane $\mathbf{6}^{6+}$ (Fig. 5) [13], specifically designed to achieve photoinduced ring shuttling. This compound is made of the electron-donor macrocycle $\mathrm{R}$, and a dumbbell-shaped component which contains (i) $\left[\mathrm{Ru}(\mathrm{bpy})_{3}\right]^{2+}$ (P) as one of its stoppers, (ii) a 4,4'-bipyridinium unit $\left(\mathrm{A}_{1}\right)$ and a 3,3'dimethyl-4,4'-bipyridinium unit $\left(\mathrm{A}_{2}\right)$ as electron accepting stations, (iii) a $p$-terphenyl-type ring system as a rigid spacer (S), and (iv) a tetraarylmethane group as the second stopper (T). The stable translational isomer of rotaxane $6^{6+}$ is the one in which the $\mathrm{R}$ component encircles the $\mathrm{A}_{1}$ unit, in keeping with the fact that this station is a better electron acceptor than the other one. The strategy devised in order to obtain the photoinduced abacus-like movement of the $\mathrm{R}$ macrocycle between the two stations $A_{1}$ and $A_{2}$, illustrated in the bottom part of Fig. 5, is based on the following four operations [13]:

(a) Destabilization of the stable translational isomer: light excitation of the photoactive unit $\mathrm{P}$ (process 1) is followed by the transfer of an electron from the excited state to the $A_{1}$ station, which is encircled by the ring $\mathrm{R}$ (process 2), with the consequent 'deactivation' of this station; such a photoinduced electron-transfer process has to compete with the intrinsic decay of *P (process 3 ).

(b) Ring displacement: the ring moves from the reduced $\mathrm{A}_{1}$ station to $\mathrm{A}_{2}$ (process 4 ), a step that has to compete with the back electron-transfer process from $\mathrm{A}_{1}{ }^{-}$(still encircled by $\mathrm{R}$ ) to the oxidized photoactive unit, $\mathrm{P}^{+}$(process 5).

(c) Electronic reset: a back electron-transfer process from the 'free' $\mathrm{A}_{1}{ }^{-}$station to $\mathrm{P}^{+}$(process 6) restores the electron-acceptor power to the $A_{1}$ station.

(d) Nuclear reset: as a consequence of the electronic reset, back movement of the ring from $\mathrm{A}_{2}$ to $\mathrm{A}_{1}$ takes place (process 7).

The crucial point for such a mechanism is indeed the favorable competition between ring displacement (process 4) and back electron transfer (process 5). While the kinetics of the relevant electron-transfer processes has been experimentally determined by laser flash photolysis (for process 5 in acetonitrile solution at $298 \mathrm{~K}, k \sim 2 \times 10^{5} \mathrm{~s}^{-1}$ ), the rate for the motion of $\mathrm{R}$ from $\mathrm{A}_{1}^{-}$to $\mathrm{A}_{2}$ has proven to be quite difficult to measure. Very recently, detailed time-resolved spectroscopic investigations have been carried out with $6^{6+}$ in the presence of a suitable species that is able to 
reduce $\mathrm{P}^{+}$before the back electron transfer from $\mathrm{A}_{1}{ }^{-}$can occur. The results suggest that in acetonitrile at room temperature the ring shuttling rate is slower than the back electron transfer, but of the same order of magnitude [14]. Therefore, light inputs can cause the occurrence of a forward and back ring movement (i. e., a full cycle) with no generation of waste products, though the efficiency will be low. In some way, rotaxane $6^{6+}$ can be considered as a 'four-stroke' linear motor powered by visible photons.

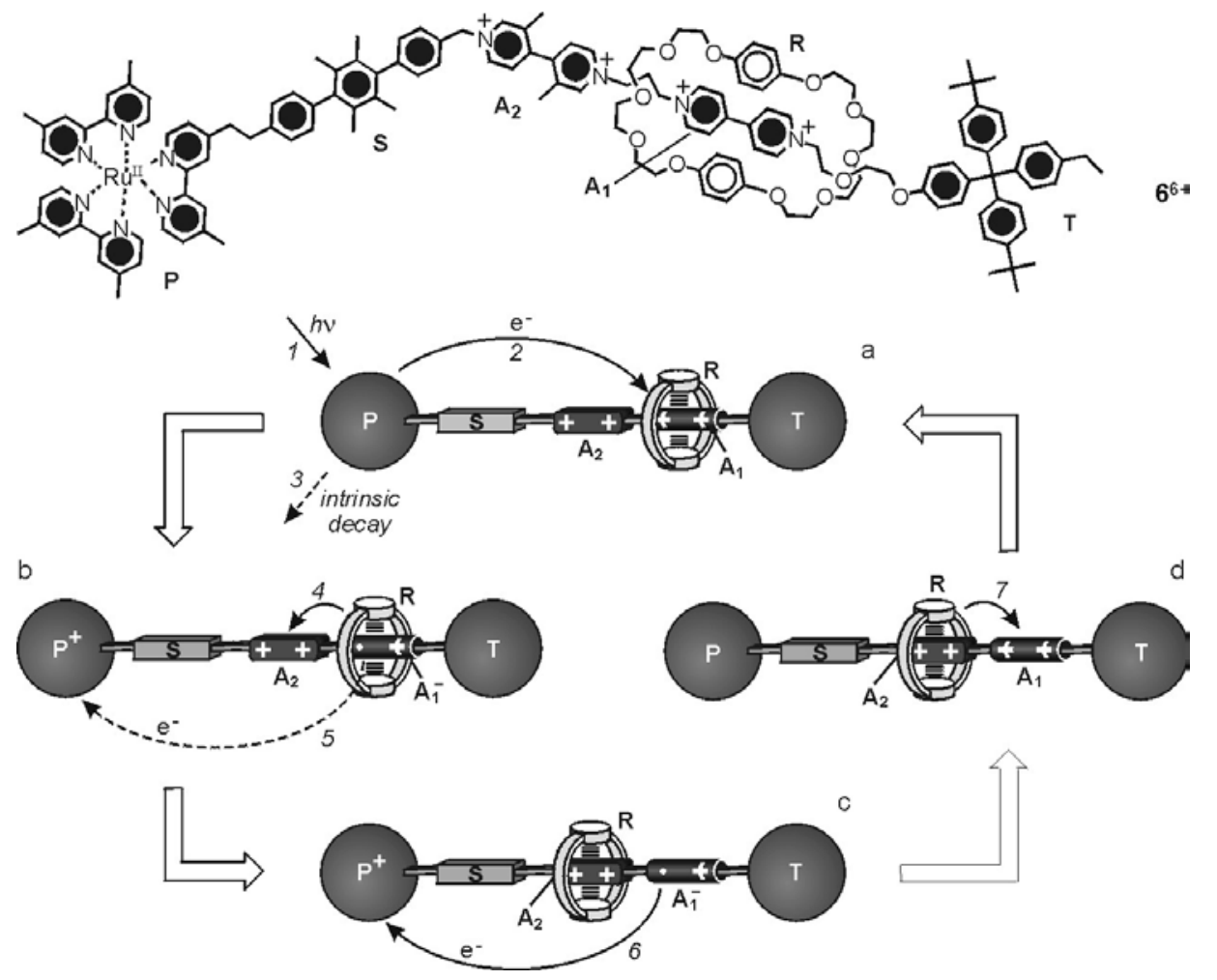

Fig. 5 Formula of rotaxane $6^{6+}$ and schematic representation of the mechanism for the photoinduced shuttling of macrocycle $\mathrm{R}$ between the two stations $\mathrm{A}_{1}$ and $\mathrm{A}_{2}$ located on the thread component.

\section{CONCLUSION}

Photoactive pseudorotaxanes and rotaxanes which behave as rudimental artificial molecular machines have been obtained using the paradigms of supramolecular chemistry. These systems constitute the first step towards the rational design and construction of molecular motors powered by light. Apart from practical applications, which are presently difficult to foresee, the extension of the concept of a machine to the molecular level is of interest not only for the development of nanotechnology, but also for the growth of basic research. Looking at supramolecular chemistry from the viewpoint of functions with references to devices of the macroscopic world is indeed a very interesting exercise which introduces novel concepts into Chemistry as a scientific discipline.

\section{ACKNOWLEDGMENTS}

We thank profs. V. Balzani, M. Venturi and the other colleagues of our group, and prof. J.F. Stoddart and coworkers for a long lasting and most profitable collaboration. Financial support 
from the European Union (STREP NMP2-CT-2003-505487 and Marie Curie Individual Fellowship HPMF-CT-2002-01916 to B.F.) and the italian MIUR (Supramolecular Devices project and FIRB RBNE019H9K) is gratefully acknowledged.

\section{REFERENCES}

1. D. S. Goodsell, Our Molecular Nature: The Body's Motors, Machines and Messages, Copernicus, New York (1996).

2. Molecular Motors, M. Schliwa (Ed.), Wiley-VCH, Weinheim (2002).

3. R.P. Feynman, Eng. Sci. 23, 22 (1960).

4. (a) V. Balzani, A. Credi and M. Venturi, Molecular Devices and Machines - A Journey into the Nano World, Wiley-VCH, Weinheim (2003); (b) Struct. Bond. 99, 1-302 (2001); (c) Acc. Chem. Res. 34, 409-522 (2001).

5. Recent prominent examples: (a) T. R. Kelly, H. De Silva and R. A. Silva, Nature 401, 150 (1999); (b) N. Koumura, R. W. J. Zijlstra, R. A. van Delden, N. Harada and B. L. Feringa, Nature 401, 152 (1999). (c) M. C. Jiménez, C. O. Dietrich-Buchecker and J.-P. Sauvage, Angew. Chem. Int. Ed. 39, 3284 (2000); (d) A. M. Brouwer, C. Frochot, F. G. Gatti, D. A. Leigh, L. Mottier, F. Paolucci, S. Roffia and G. W. H. Wurpel, Science 291, 2124 (2001); (e) D. A. Leigh, J. K. Y. Wong, F. Dehez and F. Zerbetto, Nature 424, 174 (2003); (f) J. D. Badjic, V. Balzani, A. Credi, S. Silvi and J. F. Stoddart, Science 303, 1845 (2004).

6. V. Balzani and F. Scandola, Supramolecular Photochemistry, Horwood, Chichester (1991).

7. Molecular Catenanes, Rotaxanes and Knots, J.-P. Sauvage and C. Dietrich-Buchecker (Eds.), Wiley-VCH, Weinheim (1999).

8. D. B. Amabilino and J. F. Stoddart, Chem. Rev. 95, 2725 (1995).

9. P. L. Anelli, P. R. Ashton, R. Ballardini, V. Balzani, M. Delgado, M. T. Gandolfi, T. T. Goodnow, A. E. Kaifer, D. Philp, M. Pietraszkiewicz, L. Prodi, M. V. Reddington, A. M. Z. Slawin, N. Spencer, J. F. Stoddart, C. Vicent and D. J. Williams, J. Am. Chem. Soc. 114, 193 (1992).

10. (a) R. Ballardini, V. Balzani, M. T. Gandolfi, L. Prodi, M. Venturi, D. Philp, H. G. Ricketts and J. F. Stoddart, Angew. Chem. Int. Ed. Engl. 32, 1301 (1993); (b) P. R. Ashton, R. Ballardini, V. Balzani, S. E. Boyd, A. Credi, M. T. Gandolfi, M. Gómez-López, S. Iqbal, D. Philp, J. A. Preece, L. Prodi, H. G. Ricketts, J. F. Stoddart, M. S. Tolley, M. Venturi, A. J. P. White and D. J. Williams, Chem. Eur. J. 3, 152 (1997).

11. (a) S. Chia, J. Cao, J. F. Stoddart and J. I. Zink, Angew. Chem. Int. Ed. 40, 2447 (2001). (b) R. Hernandez, H.-R. Tseng, J. W. Wong, J. F. Stoddart and J. I. Zink, J. Am. Chem. Soc. 126, 3370 (2004).

12. (a) P. R. Ashton, R. Ballardini, V. Balzani, E. C. Constable, A. Credi, O. Kocian, S. J. Langford, J. A. Preece, L. Prodi, E. R. Schofield, N. Spencer, J. F. Stoddart and S. Wenger, Chem. Eur. J. 4, 2411 (1998); (b) P. R. Ashton, V. Balzani, O. Kocian, L. Prodi, N. Spencer and J. F. Stoddart, J. Am. Chem. Soc. 120, 11190 (1998).

13. P. R. Ashton, R. Ballardini, V. Balzani, A. Credi, K. R. Dress, E. Ishow, C. J. Kleverlaan, O. Kocian, J. A. Preece, N. Spencer, J. F. Stoddart, M. Venturi, and S. Wenger, Chem. Eur. J. 6, 3558 (2000).

14. V. Balzani, A. Credi, B. Ferrer, J. F. Stoddart and M. Venturi, manuscript in preparation. 